\title{
Ileal stricturing after long-term indomethacin treatment
}

\author{
S.H. Saverymuttu, A. Thomas, A. Grundy and J.D. Maxwell \\ Departments of Medicine, Histopathology and Radiology, St. George's Hospital and Medical School, Cranmer \\ Terrace, Tooting, London SW17 0RE, UK.
}

\begin{abstract}
Summary: A patient on long-term indomethacin therapy developed intestinal obstruction due to ileal strictures. No other cause apart from indomethacin could be implicated in the pathogenesis of the stricture.
\end{abstract}

\section{Introduction}

Recent studies have demonstrated that non-steroidal anti-inflammatory drugs (NSAIDS) can increase small intestinal permeability and produce ileal inflammation in addition to perforation and bleeding (Bjarnason et al., 1984; Langman et al., 1985). The clinical significance of the ileal inflammation is uncertain since these small bowel lesions appear to be unassociated with any gut symptoms and are reversible on discontinuing NSAIDS. If the ileal inflammation behaves in a similar fashion to the inflammation found in Crohn's disease it may take several years of therapy before stricture formation develops. We report a patient with rheumatoid arthritis on long-term indomethacin therapy who developed small intestinal strictures where no other cause apart from indomethacin therapy could be implicated.

\section{Case history}

A 53 year old Indian female with an 8-year history of rheumatoid arthritis presented with a severe iron deficiency anaemia (haemoglobin $5.2 \mathrm{~g} / \mathrm{dl}$ ) with positive faecal occult bloods. She had received in domethacin $(75-150 \mathrm{mg} /$ day) for 5 years continuously together with a year's course of gold which had been discontinued 2 years previously. Although she denied any symptoms related to the small bowel, a small bowel meal demonstrated a grossly abnormal ileocaecal region. There was evidence of fissuring and stricture formation involving the terminal ileum and caecal pole, the appearances resembling Crohn's disease (Figure 1). Operation was declined and she was transfused and discharged with her arthritis therapy changed to paracetamol. Over the following 9 months

Correspondence: S.H. Saverymuttu, M.R.C.P., Department of Medicine II, St. George's Hospital Medical School, Cranmer Terrace, London SW17 0RE, UK.

Accepted: 30 April 1986 she maintained a normal haemoglobin but developed subacute intestinal obstruction. She now agreed to surgical treatment and at laparotomy two strictures were resected by a right hemicolectomy. Macroscopically the resected specimen showed areas of small bowel ulceration with intervening normal mucosa,

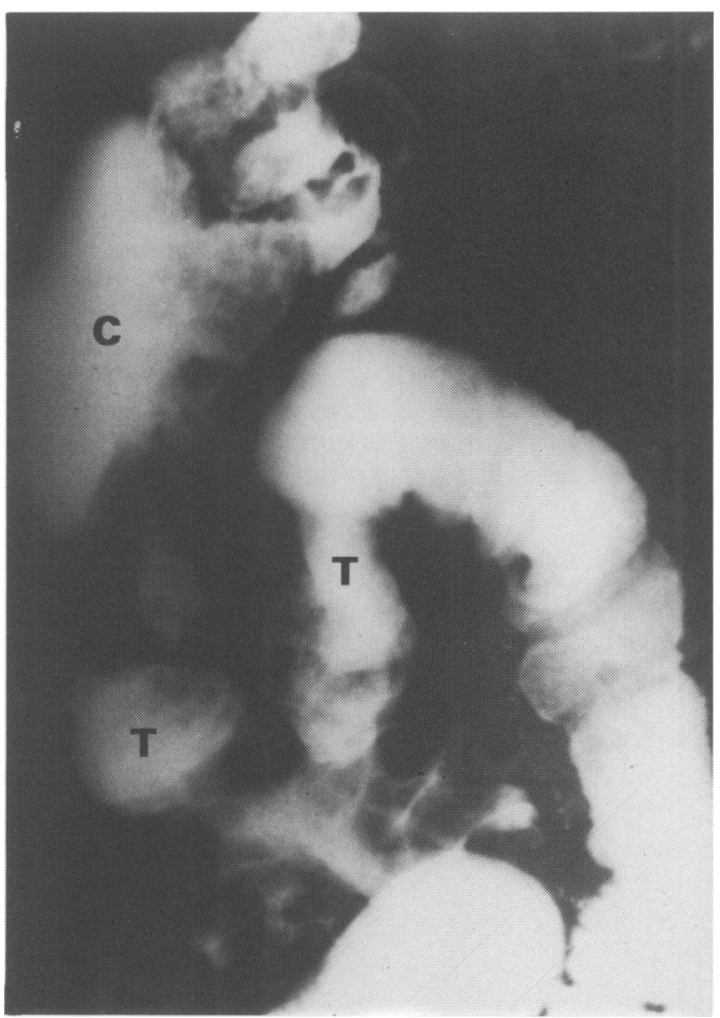

Figure 1 Small bowel meal showing grossly distorted and stenotic segment of terminal ileum (T) and lower pole of caecum (C).

(C) The Fellowship of Postgraduate Medicine, 1986 
thickening of the bowel wall and stricture formation in the terminal ileum and at the ileocaecal junction. Histology confirmed mucosal ulceration of the small bowel and showed chronic inflammation with fibrosis and loss of muscularis propria without any features suggestive of Crohn's disease, tuberculosis, ischaemic bowel disease or vasculitis. Following surgery she made an excellent recovery and over a 5-year followup period has had no recurrence of anaemia. No antituberculous treatment was given. A small bowel enema examination carried out 4 years after the hemicolectomy showed no evidence of recurrent disease in the small bowel.

\section{Discussion}

Animal studies have clearly shown that indomethacin can produce small intestinal ulcerative lesions (Kent $e t$ al. (1969). Indirect evidence for NSAID ileal inflammation in man has been provided by ${ }^{111}$ indium leucocyte scanning where ileal accumulation of labelled cells has been found after treatment with indomethacin (Bjarnason et al., 1984). More direct evidence for NSAID ileal damage in man has been scarce and reflects the difficulty in adequately visualizing the terminal ileum either radiologically or endos-

\section{References}

BJARNASON, I., SO, A., LEVI, A.J., PETERS, T.J., WILlIAMS, P., ZANELLI, G.D., GUMPEL, J.M. \& ANSELL, B. (1984). Intestinal permeability and inflammation in rheumatoid arthritis: effects of non steroidal anti-inflammatory drugs. Lancet, ii, 1171.

LANG, J., BJARNASON, I., LEVI, A.J. \& PRICE, A.B. (1985). Pathology of iatrogenic ileal strictures caused by nonsteroidal anti-inflammatory drugs. Gut, 26, A542 (Abstract). copically. Although it is impossible to prove a causal relationship between NSAIDS and ileal strictures in the present case alternative aetiologies are unlikely. There were no histological features of Crohn's disease in the resected specimen and over a 5-year follow-up period we have failed to demonstrate recurrent disease at the anastomotic site or elsewhere. An intensive search for tuberculosis was made at surgery and both cultures and histology proved negative and again there has been no evidence for reactivation over the 5-year follow-up. There was no history of potassium chloride ingestion.

Without the recent interest in NSAIDS and small bowel damage the association between indomethacin and small intestinal strictures in the present case would have gone unrecognized and been labelled as atypical Crohn's disease, healed tuberculosis or idiopathic ileal strictures. It is interesting that the preliminary report of a review of small bowel resections from a district general hospital revealed three cases of 'idiopathic ileal strictures' who had received prolonged treatment with NSAIDS (Lang et al., 1985). The magnitude of NSAIDS small bowel damage is unknown because it has been hitherto unrecognized. Increased awareness of this potentially avoidable condition should allow early recognition and appropriate management.

LANGMAN, M.J.S., MORGAN, L. \& WORRALL, A. (1985). Use of anti-inflammatory drugs by patients admitted with small or large bowel perforations and haemorrhage. British Medical Journal, $290,347$.

KENT, T.H., CARDELL, R.M. \& STAMLER, F.W. (1969). Small intestine ulcers and intestinal microflora in rats given indomethacin. American Journal of Pathology, 54, 237. 